\title{
Test of cold denaturation mechanism for proteins as a function of water's structure
}

\author{
Manuel I. Marqué:* \\ Departamento de Física de Materiales $C-I V$, \\ Universidad Autónoma de Madrid, 28049 Madrid, Spain
}

\begin{abstract}
In a recent paper [PRL 91, 138103 (2003)] a new mechanism to explain the cold denaturation of proteins, based on the loss of local low-density water structure, has been proposed. In the present paper this mechanism is tested by means of full atom numerical simulations. In good agreement with this proposal, cold denaturation resulting in the unfolded state was found at the High Density Liquid (HDL) state of water, at which the amount of open tetragonal hydrogen bonds decreases at cooling.
\end{abstract}

Hydrophobicity is an important driving force in the process of polymer folding and protein stabilization 1, 2, 3, 4, 5, 6, 7, 8, 9]. Basically, hydrophobicity provides a mechanism to avoid the energy cost of disruption of a water hydrogen bond by the presence of an apolar molecule . Actually, the folding of a polymer is partially due to the assembly of the apolar monomers in order to minimize the disruption of hydrogen bonds among water molecules [4, 8, 10]. Also, the stability of the folded state is enhanced by the cage structures formed of highly organized water molecules around the biopolymer where the disruption of hydrogen bonds is minimized [11]. Increasing the temperature of a polymer in a water bath leads to structures different from the native folded state. Hydrophobicity is reduced by thermal fluctuations and the proteins stability is lost. However it is also possible to reduce polymers hydrophobicity and to induce unfolded states by lowering the temperature, a phenomenon called cold denaturation [12, 13, 14]. The microscopic mechanism leading to cancellation of the hydrophobic effects and cold denaturation is not clear, due in part to the complexity of the protein-solvent interactions. Cold denaturation has two important properties. (i) For pure water solvent, cold denaturation is only found at very high pressures $(P>2 k b a r)$ [15]. So, in order to get high pressure cold destabilization of the folded protein, hydrophobicity should decrease when cooling at high pressures. (ii) The transition to the unfolded state is driven by a smaller volume, indicating a positive slope in the P-T transition line. On the contrary, the usual thermal denaturation behaves with negative slope. Hydrophobicity weakening at high pressures should also be accompanied by a positive slope. In recent years there has been a huge amount of work in order the study the dependence of hydrophobicity with pressure. Contact and solvent separated configurations have been studied from an enthalpy/entropy point of view [16]. Solvent separated configurations are increasingly stabilized at higher pressures by enthalpic contributions. Full atom simulations of two Lennard-Jones particles in model water have found that the aggregation of the two particles becomes unstable for pressures in the kbar range [17], and recent replica exchange molecular dynamics simulations have studied the weakening of the hydrophobic interaction with pressure for a C-terminal fragment of protein G [18]. These findings are in agreement with a cancellation of the hydrophobic force at high pressures. Actually some recent molecular dynamics simulations have already found the swelling of a protein when lowering the temperature at high pressures with a positive transition slope as corresponding to the cold dentauration of real proteins [19]. However the question still remains: Why is hydrophobicity canceled at very high pressures in such a way as to promote cold denaturation?

During the last years, several works have attempted to answer this question by using different models: Thermodynamical models [20], effective attraction models [21], temperature dependent attraction models 22], effective water-protein interactions models including solvent exclusion [7, 10, 23], lattice models 24] based on a bimodal description of the energy of water in the shell around the hydrophobic molecule 25] and models mimicking the interaction between water and non polar monomers 26]. Very recently, a lattice model that captures the effect of volume correlations on water at high and low pressures has been used to propose a possible mechanism for cold denaturation and hydrophobicity weakening at high pressures [27]. The proposal is based on the known existence of two possible water metastable states. A low density/pressure liquid with a local structure very similar to the low-density amorphous (LDA) solid water, consisting of an open tetrahedral network, and a high density/pressure water, very similar to high-density amorphous (HDA) solid water, where core-core repulsion dominates, similar to simple, non anomalous liquids [28]. A way to change from the low density liquid (LDL) to the high density liquid (HDL) is by increasing the pressure at constant temperature. Actually, there is a critical pressure $P_{c}$ separating both behaviors. For $P<P_{c}$ a decrease in temperature implies an increase in the number of energetically favorable open tetrahedral interactions, and an increase on volume. For a given temperature (called temperature of maximum density (TMD)) this increase in volume is more important than the regular 
compresion of the liquid, and the density reaches a maximum. Then, the density decreases more and more until reaching the negative sloped Ice Ih crystalline line ,or the negative sloped Kauzmann boundary (vanishing diffusivity) in numerical simulations. On the contrary for $P>P_{c}$ the number of interactions forming open tetrahedral structures decreases when cooling down the system, producing the more dense interpenetrating tetrahedral network and the more energetically dominant core-core interactions. Now there is no TMD, and the density always decreases (similar to a non-anomalous liquid) until reaching the positive sloped, Ice III or Ice V crystalline line (or the positive sloped Kauzmann boundary in numerical simulations). Basically, for a constant temperature, the higher the pressure, the lower the number of low density open tetrahedral structures. The hypothesis proposed in ref. 27] is that hydrophobicity (i.e. aggregation to avoid the disruption of hydrogen bonds and formation of open cage structures) is drastically reduced for the HDL state. Basically, at the HDL state, the existence of solvent-separated apolar molecules is not energetically unfavorable as core-repulsion interaction is now the prevailing force instead of the open tetrahedral hydrogen bonded network. Also, the stability due to the formation of low density hydrogen bonded cage structures is not energetically favorable anymore. The high density water molecules are now capable of penetrating inside the protein leading to unfolding [7]. With this hypothesis is easy to understand why cold dentauration in pure water is found at $P>2 k b a r$, since $P_{c} \sim 2 k b a r$ in pure water. Also it is possible to understand why the cold denaturation slope at the $\mathrm{P}-\mathrm{T}$ phase diagram is positive, since the non-anomalous, high pressure, ice crystalline lines (such as Ice III or Ice V) have also a positive slope (at $P>P_{c}$ the density of water always decreases when cooling down). In order to check this idea, a simple lattice model was proposed in Ref. [27] and the results were successfully compared with the ones corresponding to real proteins such as Ribonuclease A 29]. However, a direct comprobation of this hypothesis by means of "real" full atom simulations was still lacking. The aim of this paper is to present results from full atom simulations which clearly correlate the weakening of hydrophobicity to the presence of the high density state of water.

In this paper the SPC/E model of water 30] is simulated at different densities and temperatures. For positive pressures the line of temperatures of maximum density (TMD) has a negative slope in the $\mathrm{P}-\mathrm{T}$ plane and disappears for pressures $P>P_{c} \sim 300 M P a$ [31]. From the point of view of density-temperature simulations it implies that there is a critical density $\rho_{c} \sim 1.1 \mathrm{~g} / \mathrm{cm}^{3}$. If we do constant density simulations at $\rho<\rho_{c}$ we are at the LDL regime, and the number of open hydrogen bonded tetrahedral networks grows as the temperature lowers. On the contrary, if we are at $\rho>\rho_{c}$ we are at the HDL regime and the number of open hy-

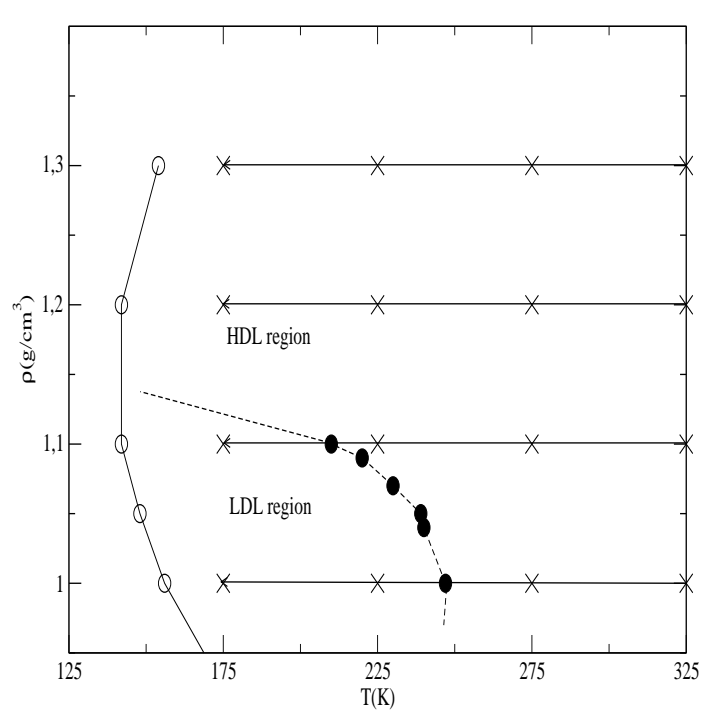

FIG. 1: Density-temperature phase diagram of SPC/E water. Black points correspond to the TMD line and white points correspond the the Kauzmann boundary 31]. Large crosses indicate the $\rho$ vs. $T$ conditions considered. Note how both regimens (LDL and HDL) are taken into account.

drogen bonded tetrahedral networks decreases as lowering the temperature. For this reason, in order to study both regimes, we perform numerical simulations at $\rho=1 \mathrm{~g} / \mathrm{cm}^{3}, 1.1 \mathrm{~g} / \mathrm{cm}^{3}, 1.2 \mathrm{~g} / \mathrm{cm}^{3}$ and $1.3 \mathrm{~g} / \mathrm{cm} 3$ (see Fig.1). At all densities, we perform simulations at $T=$ $175 K, 225 K, 275 K$ and $325 K$ in order to sample from highly thermally activated water molecules to conditions close to the Kauzmann boundary. First, in order to confirm the correctness of the density-temperatures phase points chosen for the simulations, the oxygen-oxygen pair correlation function for a sample with a maximum number of 460 water molecules has been measured. A reaction field technique [32] with a cut-off of $0.79 \mathrm{~nm}$ accounts for the long-range Coulombic interactions. Configurations were sampled by means of a non-constant step Monte Carlo algorithm. Results are shown in Fig.2. They match previously reported calculations [28] and recent experimental results 33]. At $\rho<\rho_{c}$ a maximum of the oxygen-oxygen correlation function at $r \sim 4.5 \AA$ is found. This maximum grows as we cool down the system and is typical of LDA water and open tetrahedral network structures (like IceIh). It has been also measured by numerical simulations in LDL water [28]. On the contrary, at $\rho>\rho_{c}$, the peak at $r \sim 4.5 \AA$ decreases when cooling down the system an a new peak at $r \sim 3.5 \AA$ rises up. This new peak is typical of the HDA water 33 . and interpenetrating dense tetrahedral networks (like Ice VI and Ice VII). It has also been measured by computer simulations in model water [28]. With this checking we are now confident about the range of pressures to be simulated, and we know when we are dealing with LDL and when we are dealing with HDL. 

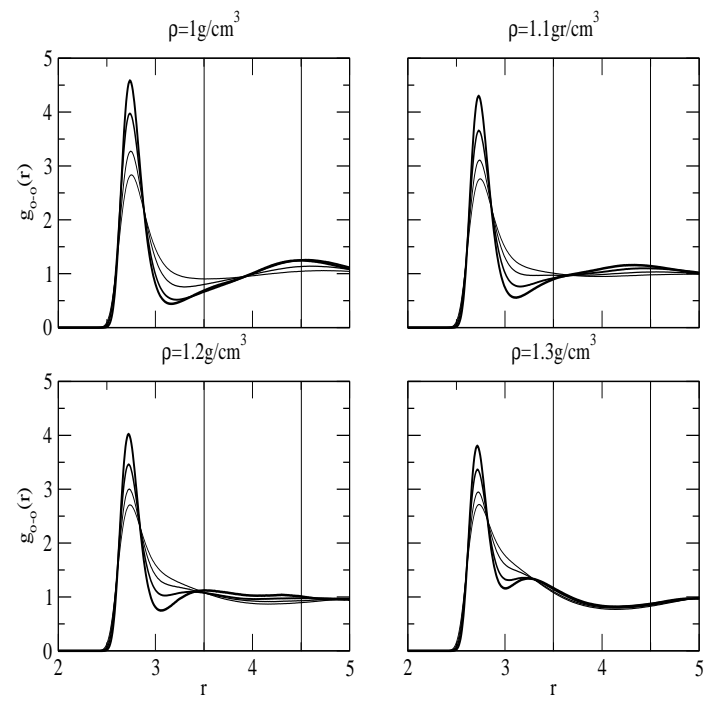

FIG. 2: Oxygen-Oxygen pair correlation functions vs. distance (in Angstroms) for the $\rho$ vs. $T$ conditions simulated in the paper. Thicker lines correspond to lower temperatures. Vertical lines indicate the values of $r$ were main secondary peaks appear: $r \sim 3.5 \AA$ for the HDL and $r \sim 4.5 \AA$ for the LDL.

To test the hypothesis proposed in Ref. 27] we should now embed an apolar polymer chain in our SPC/E water bath. If the hypothesis is correct, at low temperatures, folded states are expected for $\rho<\rho_{c}$ and unfolded states are expected for $\rho>\rho_{c}$. In order to attribute any configuration change to the influence of the solvent, we simulate a modified model of the polyethylene molecule [34], free of bond angles and dihedral potential barriers. The polymer-chain consist of $N=15$ hydrophobic $\mathrm{CH}_{2}$ beads, represented by Lennard-Jones interaction sites with $\epsilon=0.112 \mathrm{kcal} / \mathrm{mol}$ and $\sigma=1.33 \AA$ and linked to quasi-rigid harmonic bonds of $1.53 \AA$ length and interaction coupling $k_{r}=350 \mathrm{kcal} / \mathrm{mol}$. The waterpolymer cross parameters were obtained using the conventional Lorentz-Berthelot mixing rules. The structure of the hydrophobic polyethylene chain is characterized by its radius of gyration $R_{G}^{2}=1 / N \sum\left(\left(\overrightarrow{r_{i}}-r_{m c}\right)^{2}\right)$ (being $\left.r_{m c}=1 / N \sum \overrightarrow{r_{i}}\right)$. For the extended polymer the radius of gyration is $R_{G} \sim 4 \AA$ and a typical folded configuration with spherical-like symmetry has a gyration radius close to $R_{0}=2.75 \AA$. So, we consider a configuration to be folded if the gyration radius is $R_{G}<R_{0}=2.75 \AA$ (see Fig.3). For each pressure- temperature point of the phase diagram we measured up to $10^{8}$ thermally equilibrated polymer configurations in order to build a histogram with the most probable values for the gyration radius. Using these data we are able to calculate an averaged value and the corresponding dispersion. Results are shown in Fig.4 (dispersions are represented as error bars).

Clearly there are two different behaviors. For $\rho<\rho_{c}$

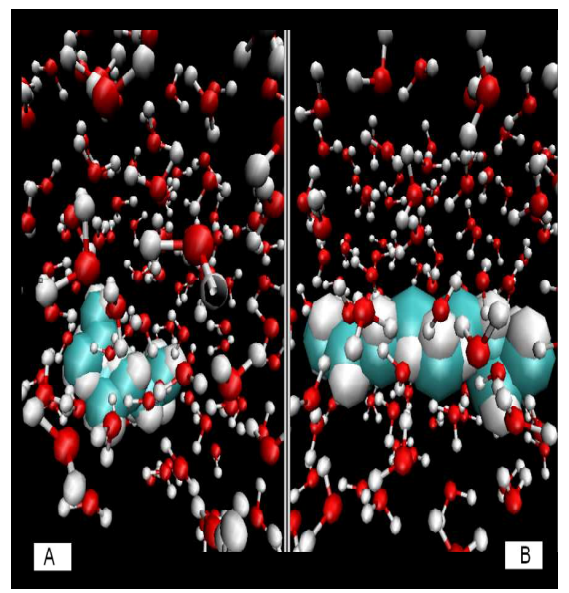

FIG. 3: (a) (Color online) Typical folded configuration of the polyethylene chain $R_{G} \sim 2 \AA$ and (b) typical unfolded configuration $R_{G} \sim 3.5 \AA$. Atom's sizes are not at scale.

and low temperatures $(T \leq 275 K)$ the protein adopts a folded state with a gyration radius $R_{G}<R_{0}$ (see Fig.4). There are no significant changes by cooling down the system. The only way to obtain unfolded states is by increasing the temperature up to $T=325 \mathrm{~K}$. In this range of densities of the system, corresponding to the LDL state, no cold denaturation is found. On the contrary when we increase the pressure up to $\rho>\rho_{c}$ (HDL state) there is a dramatic change on the protein status by decreasing the temperature (see Fig 4). At low temperatures, the gyration radius grows to a value corresponding to the unfolded state. We may definitively say that, in this range of densities of water corresponding to HDL, cold denaturation is found at low temperatures. In order to get a more direct relation between the value of the gyration radius and the water's tetragonal net status, it is possible to define a parameter $\left(S=g_{o-o}(r=4.5 \AA)-g_{o-o}(r=3.5 \AA) /\left(g_{o-o}(r=\right.\right.$ $\left.\left.4.5 \AA)+g_{o-o}(r=3.5 \AA)\right)\right)$ based on the two secondary maxima of the oxygen-oxygen pair correlation function. If the water's status corresponds to a LDL, the value of the correlation function at $r=4.5 \AA$ is going to be much important than the value of the correlation pair distribution function at $r=3.5 \AA$ (see Fig.2), and $S$ is going to be positive. If the status corresponds to a HDL, the value at $r=3.5 \AA$ is going to be larger than the value at $r=4.5 \AA$ (see Fig.2) and $S$ turns negative. Fig. 5 is a plot of the different gyration radius values found $R_{G}$ vs. $S$ (see Fig.5). The set gets divided in four quadrants: (i) $R_{G}>R_{0}$ and $S>0$ (unfolded state and LDL water),(ii) $R_{G}>R_{0}$ and $S<0$ (unfolded state and HDL water), (iii) $R_{G}<R_{0}$ and $S>0$ (folded state and LDL water), (iv) (1) $R_{G}<R_{0}$ and $S<0$ (folded state and HDL water). (In order to avoid effects on the water structure 

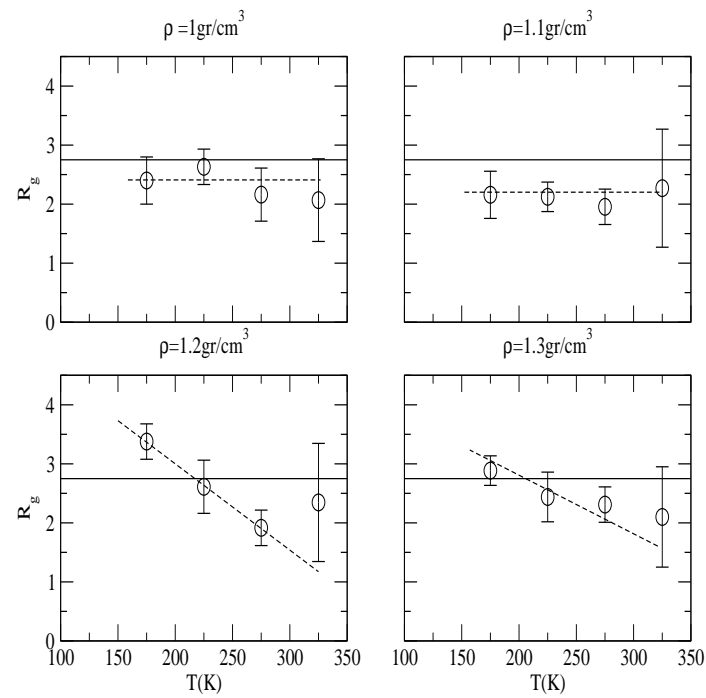

FIG. 4: Mean value and dispersion of the gyration radius (in Angstroms) for the polyethylene chain at the different $\rho$ vs. $T$ conditions considered (dotted line is a guide to the eye). Continuous line indicates the value $R_{0}=2.75 \AA$. At $\rho=$ $1 \mathrm{~g} / \mathrm{cm}^{3}, 1.1 \mathrm{~g} / \mathrm{cm}^{3}$ (LDL state) no cold denaturation is found by cooling and the gyration radius remains almost constant. At $\rho=1.2 \mathrm{~g} / \mathrm{cm}^{3}, 1.3 \mathrm{~g} / \mathrm{cm}^{3}$ (HDL state) cold denaturation is found and the gyration radius grows when cooling down.

due to thermal fluctuations we focus our attention on the low temperature regime $T<275 K)$. Note how almost all data corresponds to cases (ii) and (iii), in agreement with the hypothesis proposed in Ref. [27.

To conclude, full atom simulations of the folding behavior of a model polyethylene embedded in SPC/E water show that there is a correlation between the cold denaturation and the density of water. For LDL the existence of a local structure consisting of an open tetrahedral hydrogen bonded network prevents cold denaturation. The volume of this network increases when cooling down the system and so does hydrophobicity. On the contrary, for the high pressure HDL, the local structure is a packed tetrahedral hydrogen bonded network dominated by core-core repulsions. The volume of this network decreases when cooling down the system and so does hydrophobicity, explaining the existence of high pressure cold denaturation of proteins.

Comments from J.A.Gonzalo and H.E.Stanley are greatfully acknowledged.

* Electronic address: manuel.marques@uam.es

[1] P.L. Privalov and S.J. Gill, Adv. Protein Chem. 39, 191 (1988).

[2] N.T. Southhall, K.A. Dill and A.D.J. Haymet, J. Phys. Chem. B 106, 521 (2002).

[3] L.R. Pratt and A. Pohorille, Chem. Rev. 102, 2671

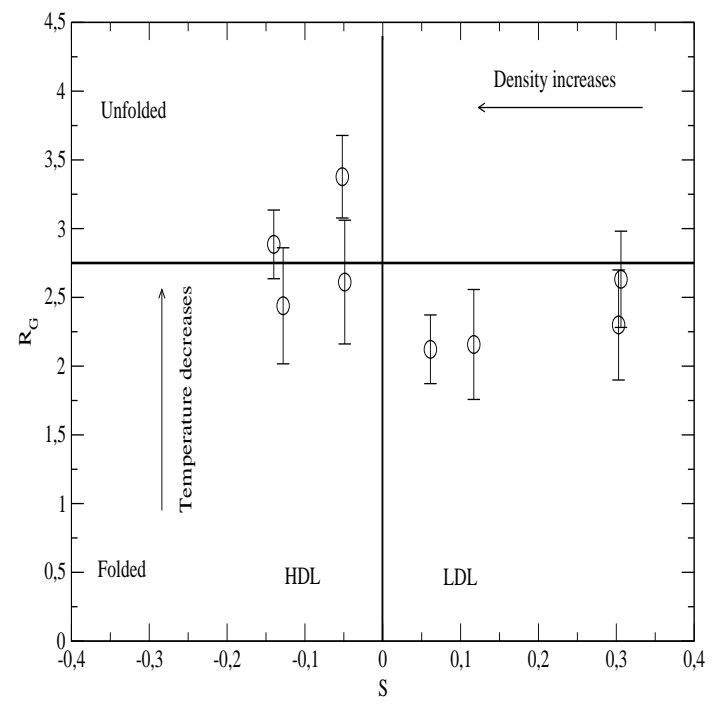

FIG. 5: Mean value and dispersion of the gyration radius (in Angstroms) vs. water's structure parameter $(S)$ (see text). Continuous horizontal line indicates a separation between folded and unfolded states. Continuous vertical line indicates the separation between the LDL and the HDL. Note how cold denaturation is mainly found at the HDL state.

(2002).

[4] W. Kauzmann, Adv. Protein Chem. 14, 1 (1959).

[5] K. A. Dill, D. O. V. Alonso and K. Hutchinson, Biochemistry 28, 5439 (1989); K. F. Lau and K. A. Dill, Macromolecules 22, 3986 (1989); K. A. Dill and D. Stigter, Adv Prot Chem 46, 59 (1995).

[6] K. A. Dill, Biochemistry 29, 7133 (1990); H. S. Chan and K. A. Dill, Phys. Today 46(2), 24 (1993).

[7] G. Hummer, S. Garde, A. E. Garcia and M. E. Paulaitis, Proc. Natl. Acad. Sci. USA 95, 1552 (1998).

[8] D. Chandler, Nature 417, 491 (2002); P. R. ten Wolde and D. Chandler, Proc. Natl. Acad. Sci. USA 99, 6539 (2002); K. Lum, D. Chandler and J. D. Weeks, J. Phys. Chem. B 103, 4570 (1999); P. R. ten Wolde, J.Phys. : Condens. Matter 14, 9445 (2002).

[9] S. Melchionna, G. Briganti, P. Londei and P. Cammarano, Phys. Rev. Lett. 92158101 (2004).

[10] F. H. Stillinger, J. Solut. Chem. 2, 141 (1973).

[11] H. S. Frank and M. W. Evans, J. Chem. Phys. 13, 507 (1945).

[12] C. N. Pace and Ch. Tanford, Biochemistry 7, 198 (1968).

[13] P. L. Privalov, Crit. Rev. Biochem. Mol. Biol. 25, 281 (1990); in T. E. Creighton, Protein Folding (W.H. Freeman, New York, 1992); G. P. Privalov and P. L. Privalov, Methods Enzymol. 323, 31 (2000).

[14] J. Jonas, ACS Symp. S 676, 310 (1997).

[15] S. Kunugi and N. Tanaka, Biochimica et Biophysica Acta 1595, 329 (2002). L. Smeller, ibid. 1595, 11 (2002).

[16] T.Ghosh, A. E. García and S. Garde, J. Am. Chem. Soc., 123, 1097 (2001); T.Ghosh, A. E. García and S. Garde, J. Chem. Phys., 116, 2480 (2002); T.Ghosh, A. E. García and S. Garde, J. Phys. Chem. B., 107, 612 (2003)

[17] V. A. Payne et al., J. Phys. Chem. B 101, 2054 (1997); S. Shimizu and H. S. Chan, J. Chem. Phys. 113, 4683 (2000). 
[18] D. Paschek and A. E. García, Phys. Rev. Lett. 93238105 (2004).

[19] D. Paschek, S. Nonn and A. Geiger, Phys. Chem. Chem. Phys. 7, 2780 (2005).

[20] S. A. Hawley, Biochemistry 10, 2436 (1971); Methods Enzymol. 49, 14 (1978); J. Zipp and W. Kauzmann, Biochemistry 12, 4217 (1973).

[21] C. Vanderzande, Lattice Models of Polymers (Cambridge University Press, Cambridge, 1998).

[22] K.A. Dill et al. Biochemistry 28, 5439 (1989); H.S. Chan and K. A. Dill, Proteins 30, 2 (1998); S. Shimizu and H.S. Chan, Proteins 48, 15 (2002).

[23] L. R. Pratt and D. Chandler, J. Chem. Phys. 673683 (1977); R. A. Pierotti, ibib. 67, 1840 (1963); M. S. Cheung, A. E. García and J. N. Onuchic, Proc. Nat. Acad. Sci. 99, 685 (2002).

[24] P. De los Rios and G. Caldarelli, Phys. Rev. E 62, 8449 (2000); Phys. Rev. E 63, 031802 (2001); G. Caldarelli and P. De los Rios, J. Biol. Phys. 27, 279 (2001); A. Hansen, M. H. Jensen, K. Sneppen and G. Zocchi, Eur. Phys. J. B 6, 157 (1998); A. Trovato, J. van Mourik and A. Marian, Eur. Phys. J. B 6, 63 (1998).

[25] N. Muller, Acc. Chem. Res 23, 23 (1990); B. Lee and G. Graziano, J. Am. Chem. Soc. 22, 5163 (1996); K. A. T. Silverstein, A. D. J. Haymet and K. A. Dill, J. Chem.
Phys. 111, 8000 (1999).

[26] P. Bruscolini and Lapo Casetti, Phys. Rev. E 61, R2208 (2000); Phys. Rev. E 64, 051805 (2001); J. Biol. Phys. 27, 243 (2001); A. Bakk, Phys. Rev. E 63, 061906 (2001); A. Bakk, J. S. Hoye, A. Hansen, Biophys. J. 82, 713 (2002); P. Bruscolini, C. Buzano, A. Pelizzola and M. Pretti, Phys. Rev. E 64, 050801 (2001); O. Collet, Europhys. Lett. 53, 93 (2001).

[27] M. I. Marqués, J. M. Borreguero, H. E. Stanley and N. Dokholyan, Phys. Rev. Lett. 91, 138103 (2003).

[28] F. W. Starr, M. C. Bellissent-Funel and H. E. Stanley, Phys. Rev. E 60, 1084 (1999); F. W. Starr, F. Sciortino and H. E. Stanley, Phys. Rev. E 60, 6757 (1999).

[29] J. Zhang et al. Biochemistry, 34, 8631 (1995).

[30] H. J. C. Berendsen, J. R. Grigera, and T. P. Stroatsma, J. Phys. Chem. 91, 6269 (1987).

[31] A. Scala, F. W. Starr, E. La Nave, H. E. Stanley and F. Sciortino, Phys. Rev. E 62, 8016 (2000).

[32] O. Steinhauser, Mol. Phys. 45, 335 (1982).

[33] J. L. Finney, A. Hallbrucker, I. Kohl, A. K. Soper and D. T. Bowron, Phys. Rev. Lett. 88225503 (2002).

[34] L. Larini, A. Barbieri, D. Prevosto, P. A. Rolla and D. Leporini, J. Phys.: Condens. Matter 17, L199 (2005). 